\title{
On the particle paths and the stagnation points in small-amplitude deep-water waves
}

\author{
Delia IONESCU-KRUSE \\ Institute of Mathematics of the Romanian Academy, \\ P.O. Box 1-764, RO-014700, Bucharest, Romania \\ E-mail: Delia.Ionescu@imar.ro
}

\begin{abstract}
In order to obtain quite precise information about the shape of the particle paths below small-amplitude gravity waves travelling on irrotational deep water, analytic solutions of the nonlinear differential equation system describing the particle motion are provided. All these solutions are not closed curves. Some particle trajectories are peakon-like, others can be expressed with the aid of the Jacobi elliptic functions or with the aid of the hyperelliptic functions. Remarks on the stagnation points of the small-amplitude irrotational deep-water waves are also made.
\end{abstract}

\section{Introduction}

The purpose of the present work is to investigate the fluid dynamics at the propagation of small-amplitude gravity waves over irrotational deep water. It was widely believed that as small-amplitude gravity waves propagate on the surface, the particles of the fluid move on circular orbits, the diameter of which decreases with depth (see, for example, [16, [30], [32, [34]). Indeed, in the first approximation, the fluid particles follow closed paths, but, by a phaseplane analysis of the nonlinear system describing the particle motion, in [12] and $[8]$ it is shown that no particle trajectory is actually closed, unless the free surface is flat, there exists a forward drift over a period which decreases with greater depth. Similar conclusions hold also within the full nonlinear framework of the water-wave problem for symmetric periodic steady gravity waves (Stokes waves) travelling over a flat bed or over water of infinite depth. For a qualitative description of the particle trajectories in this framework, by methods from the theory of harmonic functions, see [6] and 21] (see also the very recent book [7]). The results in [6] are recovered by a simpler approach in [14, where there are also described all possible particle trajectories beneath a Stokes wave. The particle trajectories change considerably according to whether the Stokes waves enter a still region of water or whether they interact with a favorable or 
adverse uniform current. Some particle trajectories are closed orbits, some are undulating paths and most are looping orbits that drift either to the right or to the left, depending on the underlying current.

Notice that there are only a few explicit solutions to the full nonlinear waterwave problems. For periodic gravity water waves in water of infinite depth, Gerstner constructed an explicit solution 1 in 1802 [19. Gerstner's wave is a two-dimensional wave given in the Lagrangian description, by following the evolution of individual water particles. The motion of the water body induced by the passage of Gerstner's wave is rotational, it occurs in a flow with a specific non-constant vorticity. The fact that this flow is very special is confirmed also by the fact that this is the only steady flow satisfying the constraint of constant pressure along the streamlines cf. 31. Beneath Gerstner's wave it is possible to have a motion of the fluid where all particles describe circles with a depthdependent radius [4, 23].

In order to obtain quite precise information about the shape of the particle paths below small-amplitude gravity waves travelling on irrotational deep water, in this paper we provide analytic solutions of the nonlinear differential equation system describing the particle motion. We show that all these solutions are not closed curves. In the study of the nonlinear system (35), a peakon-like trajectory (54) comes up. This solution has a vertical asymptote in the positive direction (see Figure 2). A peakon-like solution appeared also as particle path below small-amplitude periodic gravity waves travelling on a constant vorticity current (see [28] and 29]). The other solutions of the nonlinear system (35) are given by (69). They cannot be expressed in terms of elementary functions. In some cases these solutions can be expressed with the aid of the Jacobi elliptic functions, in other cases they can be expressed with the aid of the hyperelliptic functions. We draw some of the curves obtained for different values of the parameters (see Figure 4, Figure 5, Figure 6). We observe that some solutions (see (89)) have vertical asymptotes in the positive direction (see Figure 6 too). This surprising feature is also found at the solutions describing the motion of the particles beneath small-amplitude capillary-gravity waves which propagate on the surface of an irrotational water flow with a flat bottom (see [27]). The particle seems to be shot out from the flow, this feature could reflect the wavebreaking phenomenon.

For additional information on the particle paths within different types of progressive water waves, in the framework of linear theory or in the framework of full nonlinear theory of periodic symmetric waves, and in the presence or not of the background currents and vorticity, see the following references: [6]- 14 , [21-22], 24]-29, 36, 44].

Another remarkable feature that we investigate in this paper are the stagnation points for our problem, that is, points where the vertical component of the fluid velocity field is zero while the horizontal component equals the speed of the wave profile. The stagnation points are of special interest because they

\footnotetext{
1 This solution was independently re-discovered later by Rankine 39]. Modern detailed descriptions of this wave are given in the recent papers 4] and 23].
} 
are points where the flow characteristic often change. They could be located on the free surface, in this case the wave is called extreme wave, on the bottom or inside the fluid domain. For irrotational flows, the existence of extreme waves was predicted by Stokes [41, who also conjectured in 1880 that their profiles necessarily have corners with an angle of $120^{\circ}$ at the crest. A rigorous proof of this conjecture has not been given until 1982, when it was established independently in [1] and [38]. For rotational flows, the existence theory of smallamplitude waves is due to [17], 20], 45], while the existence of rotational waves of large amplitude (approaching flows with stagnation points) in flows without stagnation points was recently established in [13, [15. Some considerations about the possible shape of the limiting flow of the waves that almost admit a stagnation point are made in the paper [43. But in the rotational case, for small-amplitude water waves with constant vorticity the stagnation points can also occur inside the fluid domain, as shown in [18] and 29]. The papers [44] and 11] contain existence results for small-amplitude steady waves with constant vorticity in the presence of stagnation points in the flow. The stagnation points around which the fluid rotates are centers. In irrotational deep water, there were revealed in [35, although numerical and not completely rigorous (see [5]), stagnation points inside the fluid domain with discontinuous streamlines near the wave crests. These stagnation points are of saddle-point type. The formation of the stagnation points inside the flow may also be connected with a wave-breaking phenomenon in deep water; the traditional criterion for wave breaking is that horizontal water velocities in the crest must exceed the speed of the crest, see 2 where there are also reviewed different criterions, methods, measurements and laboratory studies to detect breaking events in deep water. Our solutions (54) and (69) are also used to identify the stagnation points in small-amplitude irrotational deep-water waves. For the solution (54), a stagnation point in the fluid appears only for $t \rightarrow \pm \infty$. At this point the path of the particle has a horizontal tangent and the location of this stagnation point is on the bottom $z=-\infty$. For the solution (69), the stagnation points are obtained by solving the equation (71). This equation can be solved graphically and depending on the signs and on the values of the involved parameters it can have one, two or three solutions. Which of these solutions are inside the fluid and their nature can be determined by a further study.

\section{The general problem for propagation of gravity waves in deep water}

Deep-water waves are modelled mathematically as periodic two-dimensional waves in water of infinite depth. To describe these waves we consider a cross section of the flow that is perpendicular to the crest line with Cartesian coordinates $(x, z)$, the $x$-axis being in the direction of wave propagation and the $z$-axis pointing vertically upwards. The water flow under consideration is bounded above by the free surface $z=\eta(x, t)$ and has the bottom at $z=-\infty$. The fluid is

acted on only by the constant gravitational acceleration $g$, the effects of surface 
tension being ignored. For gravity water waves, the appropriate equations of motion are Euler's equations (see [30]):

$$
\begin{gathered}
u_{t}+u u_{x}+v u_{z}=-\frac{1}{\rho} p_{x} \\
v_{t}+u v_{x}+v v_{z}=-\frac{1}{\rho} p_{z}-g,
\end{gathered}
$$

where $(u(x, z, t), v(x, z, t))$ is the velocity field of the water, $p(x, z, t)$ denotes the pressure, and $\rho(x, z, t)$ is the density. Another realistic assumption for gravity water wave problem is the incompressibility, that is, the density $\rho$ is constant (see 34]), which implies the equation of mass conservation:

$$
u_{x}+v_{z}=0
$$

The boundary conditions that define water-wave problems come in various forms. For our problem, the dynamic boundary condition expresses the fact that on the free surface the pressure is equal to the constant atmospheric pressure denoted $p_{0}$ :

$$
p=p_{0} \text { on } z=\eta(x, t) \text {. }
$$

The kinematic boundary condition expresses the fact that the same particles always form the free-water surface:

$$
v=\eta_{t}+u \eta_{x} \text { on } z=\eta(x, t)
$$

and the fact that at great depth there is practically no motion is expressed by the following boundary condition:

$$
(u, v) \rightarrow(0,0) \text { as } z \rightarrow-\infty
$$

The governing equations and the boundary conditions (11)-(5) define the boundaryvalue problem for deep-water waves.

An important category of flows is those of zero vorticity (irrotational flows), characterized by the additional equation:

$$
u_{z}-v_{x}=0
$$

In what follows we will consider this type of flow. The idealization of irrotational flow is physically relevant in the absence of non-uniform currents in the water.

For the water wave problem, there was a great interest in the regularity, or even stronger, the real-analyticity, of the solutions and particularly of the free surface. If these functions are real-analytic in some region, we can approximate them by using power series, approach used in small-amplitude wave theory. In the irrotational case, Lewy's theorem [33. shows that the free-surface must be a real-analytic curve and that the complex potential must have an analytic extension across the boundary (see [42]). Recent developments providing the regularity/real analyticity results for rotational flows were initiated in [10 for gravity water waves over a flat bed; for deep-water waves see [37. In the case of zero vorticity, the approach in [10] yields an alternative short proof of the the 
famous results obtained previously by Lewy.

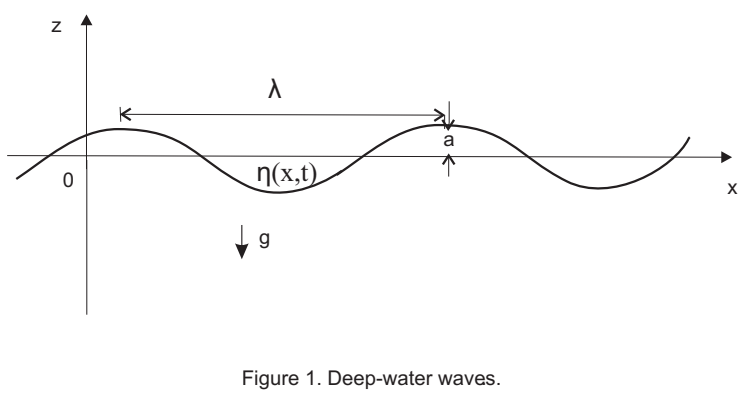

\section{Small-amplitude approximation of the water-wave problem}

Let us non-dimensionalise now the set of equations (11)-(6). If $\lambda>0$ is the wavelength and $a>0$ is the wave amplitude (see Figure 1.), we make the following change of variables (for more details see [30]),

$$
\begin{aligned}
x \mapsto \lambda x, \quad z & \mapsto z, \quad \eta \mapsto a \eta, \quad t \mapsto \frac{\lambda}{\sqrt{g}} t, \\
u & \mapsto \sqrt{g} u, \quad v \mapsto \frac{\sqrt{g}}{\lambda} v, \\
p & \mapsto p_{0}-g z+g p,
\end{aligned}
$$

where, to avoid new notation, we have used the same symbols for the nondimensional variables $x, z, \eta, t, u, v, p$ on the right-hand side. We set the constant water density $\rho=1$. Therefore, in non-dimensional variables (7), (8), the water-wave problem (11)-(6) becomes:

$$
\begin{array}{cc}
u_{t}+u u_{x}+v u_{z}=-p_{x} & \\
\frac{1}{\lambda^{2}}\left(v_{t}+u v_{x}+v v_{z}\right)=-p_{z} & \\
u_{x}+v_{z}=0 & \\
u_{z}-\frac{1}{\lambda^{2}} v_{x}=0 & \\
v=a\left(\eta_{t}+u \eta_{x}\right) & \text { on } z=a \eta(x, t) \\
p=a \eta & \text { on } z=a \eta(x, t) \\
(u, v) \rightarrow(0,0) & \text { as } z \rightarrow-\infty .
\end{array}
$$

We observe in (9) that on $z=a \eta$ both $v$ and $p$ are proportional to $a$, this being consistent with the fact that as $a \rightarrow 0$ we must have $v \rightarrow 0$ and $p \rightarrow 0$ (with no disturbance the free surface becomes a horizontal surface on which $v=p=0$ ). For consistence, one requires that $u$ is proportional to $a$ too. Thus, by the following scaling of the non-dimensional variables:

$$
p \mapsto a p, \quad u \mapsto a u, \quad v \mapsto a v,
$$


where we avoided again the introduction of a new notation, the problem (9) becomes:

$$
\begin{array}{cc}
u_{t}+a\left(u u_{x}+v u_{z}\right)=-p_{x} & \\
\frac{1}{\lambda^{2}}\left[v_{t}+a\left(u v_{x}+v v_{z}\right)\right]=-p_{z} & \\
u_{x}+v_{z}=0 & \\
u_{z}-\frac{1}{\lambda^{2}} v_{x}=0 & \\
v=\eta_{t}+a u \eta_{x} & \text { on } z=a \eta(x, t) \\
p=\eta & \text { on } z=a \eta(x, t) \\
(u, v) \rightarrow(0,0) & \text { as } z \rightarrow-\infty .
\end{array}
$$

The classical approximation is the linearized problem obtained by requiring the amplitude of the free surface to be small, that is, by letting $a \rightarrow 0, \lambda$ being fixed. Thus, we obtain the linear system:

$$
\begin{aligned}
& u_{t}+p_{x}=0 \\
& \frac{1}{\lambda^{2}} v_{t}+p_{z}=0 \\
& u_{x}+v_{z}=0 \\
& u_{z}-\frac{1}{\lambda^{2}} v_{x}=0 \\
& v=\eta_{t} \quad \text { on } z=0 \\
& p=\eta \quad \text { on } z=0 \\
& (u, v) \rightarrow(0,0) \quad \text { as } z \rightarrow-\infty \text {. }
\end{aligned}
$$

From the third equation and the forth equation in (12), we get that

$$
v_{z z}+\frac{1}{\lambda^{2}} v_{x x}=0 .
$$

Applying the method of separation of variables, we seek the solution of the equation (13) in the form

$$
v(x, z, t)=F(x, t) G(z, t) .
$$

Substituting (14) into the equation (13), we find

$$
F \frac{\partial^{2} G}{\partial z^{2}}+\frac{1}{\lambda^{2}} G \frac{\partial^{2} F}{\partial x^{2}}=0
$$

thus,

$$
\frac{1}{G} \frac{\partial^{2} G}{\partial z^{2}}=-\frac{1}{\lambda^{2}} \frac{1}{F} \frac{\partial^{2} F}{\partial x^{2}} .
$$

We observe in the above equation that the left hand side does not depend on $z$ and the right hand side does not depend on $x$. Therefore, each side must be a constant, say

$$
\frac{1}{F} \frac{\partial^{2} F}{\partial x^{2}}=-K^{2}, \quad \frac{1}{G} \frac{\partial^{2} G}{\partial z^{2}}=\frac{K^{2}}{\lambda^{2}}
$$

where $K \geq 0$ is a constant that might depend on time. With the above choice, the solutions of the equations in (17) are

$$
\begin{aligned}
F(x, t) & =\mathcal{A} \sin (K x)+\mathcal{B} \cos (K x) \\
G(z, t) & =\mathcal{C} e^{\frac{K}{\lambda} z}+\mathcal{D} e^{-\frac{K}{\lambda} z}
\end{aligned}
$$


where $\mathcal{A}, \mathcal{B}, \mathcal{C}, \mathcal{D}$ are constants depending on time. From the last condition in (12), that is, $v \rightarrow 0$ as $z \rightarrow-\infty$, we get that $\mathcal{D}=0$, and thus,

$$
v(x, z, t)=\mathcal{C} e^{\frac{K}{\lambda} z}[\mathcal{A} \sin (K x)+\mathcal{B} \cos (K x)] .
$$

On $z=0$, by the fifth equation in (12), we have $v=\eta_{t}$, which yields

$$
\mathcal{C}[\mathcal{A} \sin (K x)+\mathcal{B} \cos (K x)]=\eta_{t} .
$$

Therefore,

$$
v(x, z, t)=e^{\frac{K}{\lambda} z} \eta_{t} .
$$

Taking into account (20) and the forth equation in (12), we get

$$
u(x, z, t)=\frac{1}{K \lambda} e^{\frac{K}{\lambda} z} \eta_{t x}+\mathcal{F}(x, t),
$$

$\mathcal{F}(x, t)$ being an arbitrary function. The last condition in (12), that is, $u \rightarrow 0$ as $z \rightarrow-\infty$, yields

$$
\mathcal{F}(x, t)=0 .
$$

The components $u$ and $v$ of the velocity have to fulfill also the third equation in (12). Replacing (20), (21) with (22), in this equation, we obtain the equation that $\eta$ has to fulfill:

$$
\eta_{t x x}+K^{2} \eta_{t}=0 .
$$

We seek periodic travelling wave solutions; thus, for the equation (23) with

$$
K:=2 \pi,
$$

we choose the following solution

$$
\eta(x, t):=\cos (2 \pi(x-c t)),
$$

where $c$ represents the non-dimensional speed of propagation of the linear wave and is to be determined. With (24) and (25) in view, the components of the velocity field become

$$
\begin{aligned}
& u(x, z, t)=\frac{2 \pi c}{\lambda} e^{\frac{2 \pi}{\lambda} z} \cos (2 \pi(x-c t)) \\
& v(x, z, t)=2 \pi c e^{\frac{2 \pi}{\lambda} z} \sin (2 \pi(x-c t)) .
\end{aligned}
$$

In order to find the expressions of the pressure we take into account the first two equations in (12) and the expressions of the velocity field from above. Thus, we obtain

$$
p(x, z, t)=\frac{2 \pi c^{2}}{\lambda} e^{\frac{2 \pi}{\lambda} z} \cos (2 \pi(x-c t))+c_{0}
$$

where $c_{0}$ is a constant. On $z=0$ the pressure (27) has to fulfill the sixth equation of system (12). Hence, in view of (25), we get

$$
\frac{2 \pi c^{2}}{\lambda} e^{\frac{2 \pi}{\lambda} z} \cos (2 \pi(x-c t))+c_{0}=\cos (2 \pi(x-c t)) .
$$


The above relation must hold for all values $x \in \mathbf{R}$; therefore, we obtain

$$
c_{0}=0,
$$

and we provide the non-dimensional speed of the linear wave

$$
c^{2}=\frac{\lambda}{2 \pi}
$$

We observe that the speed of the linear wave is proportional to the square root of the wavelength. In other words, long water waves travel faster than shorter waves.

Summing up, the system (12) has the solution

$$
\begin{aligned}
& \eta(x, t)=\cos (2 \pi(x-c t)) \\
& u(x, z, t)=\frac{2 \pi c}{\lambda} e^{\frac{2 \pi}{\lambda} z} \cos (2 \pi(x-c t)) \\
& v(x, z, t)=2 \pi c e^{\frac{2 \pi}{\lambda} z} \sin (2 \pi(x-c t)) \\
& p(x, z, t)=e^{\frac{2 \pi}{\lambda} z} \cos (2 \pi(x-c t)),
\end{aligned}
$$

with $c$ given by (30).

Taking into account (7), (8), (10), we return to the original physical variables. The speed of the wave (30) and the solution (31) become:

$$
\begin{gathered}
c= \pm \sqrt{g} \sqrt{\frac{\lambda}{2 \pi}}= \pm \sqrt{\frac{g}{k}} \\
\eta(x, t)=a \cos (k(x-c t)) \\
p(x, z, t)=p_{0}-g z+a g e^{k z} \cos (k(x-c t)) \\
u(x, z, t)=a c k e^{k z} \cos (k(x-c t)) \\
v(x, z, t)=a c k e^{k z} \sin (k(x-c t)),
\end{gathered}
$$

where

$$
k:=\frac{2 \pi}{\lambda}
$$

is the wave number. The sign minus in (32) indicates a left-going wave. We observe that the velocity components have the same amplitude $a c k e^{k z}$ which depends on position and decreases exponentially with the distance below the surface.

\section{Particle trajectories}

Let $(x(t), z(t))$ be the path of a particle in the fluid domain, with location $(x(0), z(0)):=\left(x_{0}, z_{0}\right)$ at time $t=0$. The motion of the particles below the 
small-amplitude deep-water waves with the velocity field (33), is described by the following differential system:

$$
\left\{\begin{array}{l}
\frac{d x}{d t}=u(x, z, t)=A e^{k z} \cos (k(x-c t)) \\
\frac{d z}{d t}=v(x, z, t)=A e^{k z} \sin (k(x-c t)),
\end{array}\right.
$$

where the constant $A$ is

$$
A:=a c k \neq 0 .
$$

The sign of $A$ depends on the sign of the wave speed $c$. Thus, if we choose in (32) the square root with minus, that is, we consider left-going waves, we have $A<0$ and if we choose in (32) the square root with plus, that is, we consider right-going waves, we get $A>0$.

To study the exact solution of the system (35) it is more convenient to rewrite it in the following moving frame

$$
X=k(x-c t), \quad Z=k z .
$$

This transformation yields

$$
\left\{\begin{array}{l}
\frac{d X}{d t}=k A e^{Z} \cos (X)-k c \\
\frac{d Z}{d t}=k A e^{Z} \sin (X)
\end{array}\right.
$$

We write the second equation of this system in the form

$$
e^{-Z} d Z=k A \sin X(t) d t
$$

Integrating, we get

$$
-e^{-Z}=\int k A \sin X(t) d t
$$

If

$$
\int k A \sin X(t) d t<0
$$

then,

$$
Z(t)=-\log \left[-\int k A \sin X(t) d t\right]
$$

We denote by

$$
w=w(t):=\int k A \sin X(t) d t .
$$

With (41) in view, we have

$$
w<0 \text {. }
$$

From (43) we get

$$
k A \sin X(t)=\frac{d w}{d t} .
$$


Differentiating with respect to $t$ this relation, we obtain

$$
k A \cos (X) \frac{d X}{d t}=\frac{d^{2} w}{d t^{2}} .
$$

From (45) we have furthermore:

$$
k^{2} A^{2} \cos ^{2}(X)=k^{2} A^{2}-\left(\frac{d w}{d t}\right)^{2} .
$$

Thus, taking into account (42), (43), (46) and (47), the first equation of the system (38) becomes

$$
\frac{d^{2} w}{d t^{2}}=\left(-\frac{1}{w}\right)\left[k^{2} A^{2}-\left(\frac{d w}{d t}\right)^{2}\right]-k c \sqrt{k^{2} A^{2}-\left(\frac{d w}{d t}\right)^{2}}
$$

We make the following substitution

$$
\xi^{2}(w):=k^{2} A^{2}-\left(\frac{d w}{d t}\right)^{2}
$$

$A$ being different from zero by (36). Differentiating with respect to $t$ this relation, we get

$$
\xi \frac{d \xi}{d w}=-\frac{d^{2} w}{d t^{2}}
$$

We replace (49), (50) into the equation (48) and we obtain the equation

$$
\xi \frac{d \xi}{d w}=\frac{1}{w} \xi^{2}+k c \xi
$$

A solution of the equation (51) is

$$
\xi=0
$$

which, in view of (49) and (45) implies

$$
\sin X(t)= \pm 1
$$

Therefore, from (42) with the condition (41), and further from (37), a solution of the system (35) is

$$
\begin{aligned}
& x(t)=c t+\text { const }_{1} \\
& z(t)=-\frac{1}{k} \log \left(\mid k A t+\text { const }_{2} \mid\right),
\end{aligned}
$$

const $_{1}$ and const $_{2}$ are constants determined by the initial conditions $(x(0), z(0)):=$ $\left(x_{0}, z_{0}\right)$. We observe that

$$
\begin{aligned}
& \lim _{t \rightarrow-\frac{\text { const }_{2}}{k A}} x(t)=\text { const, } \\
& \lim _{\substack{t \rightarrow-\frac{\text { const }_{2}}{k A} \\
t>-\frac{\text { const }}{k A}}} z(t)=\lim _{\substack{t \rightarrow-\frac{\text { const }_{2}}{k A} \\
t<-\frac{\text { const }_{2}}{k A}}} z(t)=+\infty,
\end{aligned}
$$


and

$$
\lim _{t \rightarrow \pm \infty} x(t)= \pm \infty, \quad \lim _{t \rightarrow \pm \infty} z(t)=-\infty
$$

Therefore, $x=$ const will be a vertical asymptote and $z=-\infty$ will be a horizontal asymptote for the curve (54). The particle seems to be shot out from the flow, this feature could reflect the wave-breaking phenomenon. For $c>0$, the graph of the parametric curve (54) is drawn in the Figure 2. For $c<0$, the arrows will change the direction. This peakon-like solution appeared also as particle path below small-amplitude periodic gravity waves travelling on a constant vorticity current (see [28] and [29]).

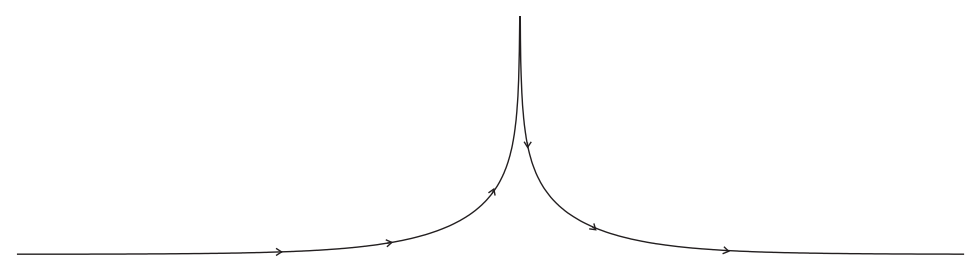

Figure 2. Peakon-like trajectory.

Calculating the derivatives of $x(t)$ and $z(t)$ with respect to $t$, we get

$$
\begin{aligned}
& x^{\prime}(t)=c \\
& z^{\prime}(t)=-\frac{A}{k A t+\text { const }_{2}} .
\end{aligned}
$$

Hence, for the solution (54) a stagnation point in the fluid, where $x^{\prime}(t)=c$, $z^{\prime}(t)=0$, appears only for $t \rightarrow \pm \infty$. We observe that at this point the path of the particle has a horizontal tangent and is located on the bottom $z=-\infty$.

The other solutions of the equation (51) satisfy

$$
\frac{d \xi}{d w}=\frac{\xi}{w}+k c
$$

The homogeneous equation

$$
\frac{d \xi}{\xi}=\frac{d w}{w}
$$

has the solution

$$
\xi(w)=\theta w,
$$

where $\theta$ is an integration constant. By the method of variation of constants, the general solution of the non-homogeneous equation (58) is given by

$$
\xi(w)=\theta(w) w,
$$

where $\theta(w)$ is a continuous function which satisfies the equation

$$
\frac{d \theta}{d w}=\frac{k c}{w} .
$$


With (44) in view, the solution of the equation (62) is

$$
\theta(w)=k c \log (-w)+\beta,
$$

$\beta$ being a constant. Therefore, the solution of the non-homogeneous equation (58) has the expression

$$
\xi(w)=w[k c \log (-w)+\beta] .
$$

Further, taking into account (49), we get the equation that $w(t)$ has to fulfill:

$$
\left(\frac{d w}{d t}\right)^{2}=k^{2} A^{2}-w^{2}[k c \log (-w)+\beta]^{2} .
$$

From (40) and (43), between $w(t)$ and $Z(t)$ there exists the following relation:

$$
w(t)=-e^{-Z(t)} .
$$

Therefore, we obtain from (65) the differential equation that $Z(t)$ has to satisfy:

$$
\left(\frac{d Z}{d t}\right)^{2}=k^{2} A^{2} e^{2 Z}-[k c Z-\beta]^{2} .
$$

By separating the variables, we get

$$
\pm \frac{d Z}{\sqrt{k^{2} A^{2} e^{2 Z}-[k c Z-\beta]^{2}}}=d t .
$$

Thus, taking into account (39) and (37), the other solution of the system (35) has the following expression:

$$
\begin{aligned}
& x(t)=c t+\frac{1}{k} \arcsin \left[\frac{1}{k A} e^{\left.-Z(t) \frac{d Z(t)}{d t}\right]}\right. \\
& \stackrel{68}{=} c t \pm \frac{1}{k} \arcsin \left[\sqrt{1-\left[\frac{k c Z(t)-\beta}{k A e^{Z(t)}}\right]^{2}}\right] \\
& z(t)=\frac{1}{k} Z(t)
\end{aligned}
$$

$Z(t)$ being the solution of the equation (68). The constant $\beta$ is determined by the initial conditions $(x(0), z(0)):=\left(x_{0}, z_{0}\right)$.

We observe that the solutions (69) are not closed curves.

Indeed, if there exists $t_{2}>t_{1}$ such that $Z\left(t_{2}\right)=Z\left(t_{1}\right)$, then, we get $z\left(t_{2}\right)=z\left(t_{1}\right)$ and $x\left(t_{2}\right)-x\left(t_{1}\right)=c\left(t_{2}-t_{1}\right) \neq 0$. If $c>0$, the particles which follow these curves will have a forward drift, if $c<0$, they will have a backward drift. $\square$

Let us make some remarks on the stagnation points inside the fluid. Calculating the derivatives with respect to $t$ of $x(t)$ and $z(t)$ from (69), we get

$$
\begin{aligned}
& x^{\prime}(t)=c+\frac{1}{k} \frac{\frac{d^{2} Z}{d t^{2}}-\left(\frac{d Z}{d t}\right)^{2}}{\sqrt{k^{2} A^{2} e^{2 Z}-\left(\frac{d Z}{d t}\right)^{2}}} \\
& z^{\prime}(t)=\frac{1}{k} \frac{d Z}{d t} .
\end{aligned}
$$


With (68) in view, for those $Z$ satisfying the following equation

$$
\left|k A e^{Z}\right|=|k c Z-\beta|,
$$

we have

$$
\frac{d Z}{d t}=0, \quad \frac{d^{2} Z}{d t^{2}}=0,
$$

and thus, $x^{\prime}(t), z^{\prime}(t)$ from (70) becomes

$$
x^{\prime}(t)=c, \quad z^{\prime}(t)=0 .
$$

Hence, for the solution (69), the stagnation points in the fluid are obtained by solving the equation (71).

The equation (71) can be solved graphically. Depending on the signs and on the values of the parameters $\mathrm{k}, \mathrm{c}, \mathrm{A}$ and $\beta$, the equation (71) can have one, two or three solutions. See, for example, in Figure 3 some possibilities that can occur. With continuous line we have drawn $\left|k A e^{Z}\right|$. Which of these solutions are inside the fluid and their nature can be determined by a further study.
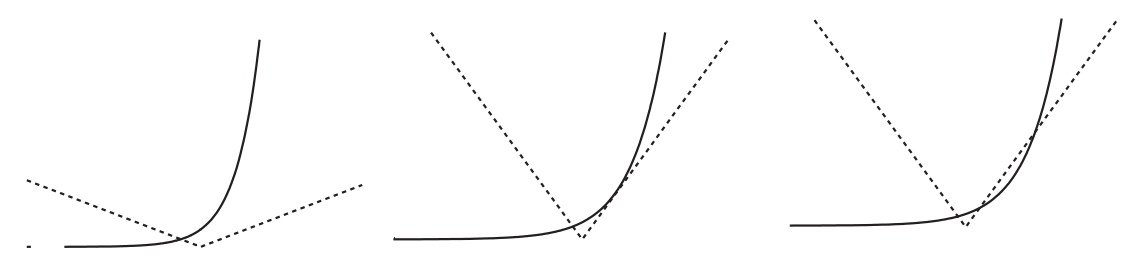

Figure 3. Graphical solutions of the equation (4.37).

Let us now investigate more the equation (68). Taking into account the expression of $e^{2 Z}$ as Taylor series:

$$
e^{2 Z}=\sum_{n=0}^{\infty} \frac{(2 Z)^{n}}{(n) !}=1+2 Z+2 Z^{2}+\frac{4 Z^{3}}{3}+\frac{2 Z^{4}}{3}+\frac{4 Z^{5}}{15}+\frac{4 Z^{6}}{45}+\cdots
$$

we get under the square root in (68) the following power series

$$
\begin{aligned}
& \left(k^{2} A^{2}-\beta^{2}\right)+2 k\left(k A^{2}+\beta c\right) Z+k^{2}\left(2 A^{2}-c^{2}\right) Z^{2}+ \\
& \quad+\frac{4 k^{2} A^{2} Z^{3}}{3}+\frac{2 k^{2} A^{2} Z^{4}}{3}+\frac{4 k^{2} A^{2} Z^{5}}{15}+\frac{4 k^{2} A^{2} Z^{6}}{45}+\cdots
\end{aligned}
$$

The constant $A$ being different from zero (36), the power series (75) contains for sure powers of $Z$ higher than two. A partial sum of the above series is a polynomial of degree higher than two, therefore, in general, $Z(t)$ cannot be expressed in terms of elementary functions. If in a partial sum of this series we consider powers of $Z$ smaller or equal than four, then, the solution of the 
equation (68) involves an elliptic integral (for elliptic integrals see, for example, [3]). Its inversion would lead to an elliptic function. If in a partial sum of the series (75) we consider powers of $Z$ higher than four, then, the solution of the equation (68) involves a hyperelliptic integral (for hyperelliptic integrals see, for example, [3, page 252). Its inversion would lead to a hyperelliptic function.

If in (75) we consider powers of $Z$ till three, then, the solution of the equation (68) involves the following elliptic integral of the first kind:

$$
\pm \int \frac{d Z}{\sqrt{\frac{4 k^{2} A^{2}}{3} Z^{3}+k^{2}\left(2 A^{2}-c^{2}\right) Z^{2}+2 k\left(k A^{2}+\beta c\right) Z+\left(k^{2} A^{2}-\beta^{2}\right)}}=t .
$$

This elliptic integral of the first kind may by reduced to the Legendre normal form.

Case 1: all the zeroes of the cubic polynomial under the square root in (76) are real and distinct. We denote them by $Z_{1}<Z_{2}<Z_{3}$. We introduce the variable $\varphi$ by (see [40] Ch. VI, $\S 4$, page 602)

$$
Z=Z_{2} \sin ^{2} \varphi+Z_{1} \cos ^{2} \varphi
$$

and we get

$$
\begin{aligned}
& \frac{4 k^{2} A^{2}}{3}\left(Z-Z_{1}\right)\left(Z-Z_{2}\right)\left(Z-Z_{3}\right)= \\
& \quad=\frac{4 k^{2} A^{2}}{3} \sin ^{2} \varphi \cos ^{2} \varphi\left(Z_{2}-Z_{1}\right)^{2}\left(Z_{3}-Z_{1}\right)\left(1-k_{1}^{2} \sin ^{2} \varphi\right)>0 \\
& d Z=2 \sin \varphi \cos \varphi\left(Z_{2}-Z_{1}\right) d \varphi,
\end{aligned}
$$

where the constant $0<k_{1}^{2}<1$ is given by

$$
k_{1}^{2}:=\frac{Z_{2}-Z_{1}}{Z_{3}-Z_{1}} .
$$

Therefore we obtain the Legendre normal form of the integral in (76):

$$
\frac{1}{\mathcal{C}_{1}} \int \frac{d \varphi}{\sqrt{1-k_{1}^{2} \sin ^{2} \varphi}}=t
$$

the constant factor in front of the integral being equal to

$$
\mathcal{C}_{1}:= \pm \frac{1}{\sqrt{3}} k|A| \sqrt{Z_{3}-Z_{1}} .
$$

The inverse of the integral in (79) is the Jacobi elliptic function sine amplitude sn (see, for example, [3])

$$
\operatorname{sn}\left(\mathcal{C}_{1} t ; k_{1}\right):=\sin \varphi .
$$

In view of the notation (77), we get

$$
Z(t)=Z_{2} \operatorname{sn}^{2}\left(\mathcal{C}_{1} t ; k_{1}\right)+Z_{1} \mathrm{cn}^{2}\left(\mathcal{C}_{1} t ; k_{1}\right),
$$


cn being the Jacobi elliptic function cosine amplitude (see, for example, [3]). We introduce (82) in (69) and we get $x(t)$ and $z(t)$ explicitly.

Case 2: the cubic polynomial under the square root in (76) has only one real solution denoted $Z_{0}$. We denote by $p$ and $q$ the real coefficients such that

$$
\begin{aligned}
\frac{4 k^{2} A^{2}}{3} Z^{3} & +k^{2}\left(2 A^{2}-c^{2}\right) Z^{2}+2 k\left(k A^{2}+\beta c\right) Z+\left(k^{2} A^{2}-\beta^{2}\right)= \\
& =\frac{4 k^{2} A^{2}}{3}\left(Z-Z_{0}\right)\left(Z^{2}+p Z+q\right) .
\end{aligned}
$$

We introduce the variable $\psi$ by (see [40] Ch. VI, $\S 4$, page 602)

$$
Z=Z_{0}+\sqrt{Z_{0}^{2}+p Z_{0}+q} \tan ^{2} \frac{\psi}{2},
$$

and we get

$$
\begin{aligned}
& \frac{4 k^{2} A^{2}}{3}\left(Z-Z_{0}\right)\left(Z^{2}+p Z+q\right)= \\
& =\frac{4 k^{2} A^{2}}{3}\left(\sqrt{Z_{0}^{2}+p Z_{0}+q}\right)^{3} \frac{\tan ^{2} \frac{\psi}{2}}{\cos ^{4} \frac{\psi}{2}}\left(1-k_{2}^{2} \sin ^{2} \varphi\right)>0 \\
& d Z=\sqrt{Z_{0}^{2}+p Z_{0}+q} \frac{\tan \frac{\psi}{2}}{\cos ^{2} \frac{\psi}{2}} d \psi
\end{aligned}
$$

where the constant $0<k_{2}^{2}<1$ is given by

$$
k_{2}^{2}:=\frac{1}{2}\left(1-\frac{Z_{0}+\frac{p}{2}}{\sqrt{Z_{0}^{2}+p Z_{0}+q}}\right) .
$$

Therefore we obtain the Legendre normal form of the integral in (76):

$$
\frac{1}{\mathcal{C}_{2}} \int \frac{d \psi}{\sqrt{1-k_{2}^{2} \sin ^{2} \psi}}=t,
$$

the constant factor in front of the integral being equal to

$$
\mathcal{C}_{2}:= \pm \frac{2}{\sqrt{3}} k|A|\left(Z_{0}^{2}+p Z_{0}+q\right)^{\frac{1}{4}} .
$$

The inverse of the integral in (86) is

$$
\operatorname{sn}\left(\mathcal{C}_{2} t ; k_{2}\right)=\sin \psi
$$

Taking into account (84), we get

$$
Z(t)=Z_{0}+\sqrt{Z_{0}^{2}+p Z_{0}+q} \frac{1-\operatorname{cn}\left(\mathcal{C}_{2} t ; k_{2}\right)}{1+\operatorname{cn}\left(\mathcal{C}_{2} t ; k_{2}\right)}
$$


We introduce (89) in (69) and we get $x(t)$ and $z(t)$ explicitly.

We observe that for that $t$ 's, denoted $\tilde{t}+\mathcal{K}$, with $\mathcal{K}$ a period, for which the periodic Jacobi elliptic function cn satisfies the equation

$$
1+\operatorname{cn}\left(\mathcal{C}_{2} t ; k_{2}\right)=0
$$

we have, in view of (89) and (69), that

$$
\lim _{t \rightarrow(\tilde{t}+K)} x(t)=\text { const }:=\tilde{x}+K, \quad \lim _{t \rightarrow(\tilde{t}+K)} z(t)=\infty
$$

Therefore, $x=\tilde{x}+K$ will be vertical asymptotes in the positive direction. This surprising feature is also found at the solutions describing the motion of the particles beneath small-amplitude capillary-gravity waves which propagate on the surface of an irrotational water flow with a flat bottom (see [27]). The particle seems to be shot out from the flow, this feature could reflect the wave-breaking phenomenon.

Let us draw below some of the curves obtained for different values of the parameters, using Mathematica 2 .

We consider $k=1, g=9.8, a=0.1$ and $\beta=1$. Then, by (32), choosing the square root with sign plus, and by (36), we get $c=3.1305, A=0.31305$. In this case, all the roots of the cubic polynomial under the square root in (76) are real and we get $Z(t)$ in the form (82). The graph of the curve obtained is drawn in Figure 4.

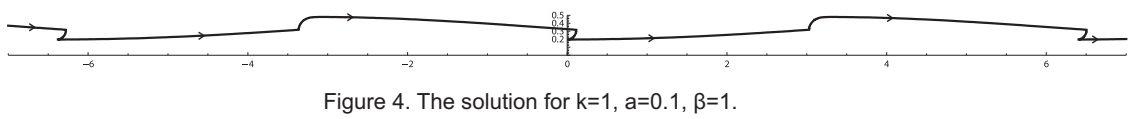

For $k=2, g=9.8, a=0.1$ and $\beta=-1$, choosing the square root with sign plus in (32), we get $c=2.21359, A=0.442718$, all the roots of the cubic polynomial under the square root in (176) are real, and thus, $Z(t)$ has the form (82). The graph of the curve obtained is presented in Figure 5.

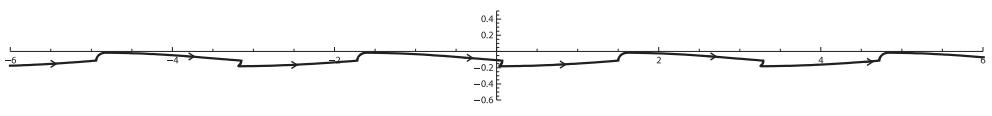

Figure 5. The solution for $k=2, a=0.1, \beta=-1$.

We choose now $k=4, g=9.8, a=0.1$ and $\beta=1$. We get by (32), choosing the square root with sign plus, $c=1.56525, A=0.6261$, but the cubic polynomial under the square root in (76) has only one real root and we get $Z(t)$ in the form (89). The graph of the curve obtained is depicted in Figure 6. We observe that this solution has vertical asymptotes in the positive direction.

\footnotetext{
${ }^{2}$ In Mathematica the Jacobi elliptic functions are implemented as JacobiSN $[u, m:=$ $\left.k_{1}^{2}\right]:=\operatorname{sn}\left(u ; k_{1}\right), \operatorname{JacobiCN}\left[u, m:=k_{1}^{2}\right]:=\operatorname{cn}\left(u ; k_{1}\right), \operatorname{JacobiDN}\left[u, m:=k_{1}^{2}\right]:=\operatorname{dn}\left(u ; k_{1}\right)$
} 


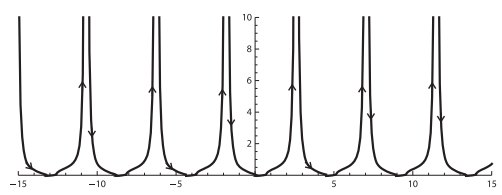

Figure 6 . The solution for $\mathrm{k}=4, \mathrm{a}=0.1, \beta=1$.

\section{References}

[1] C. J. Amick, L. E. Fraenkel and J. F. Toland, On the Stokes conjecture for the wave of extreme form, Acta Math., 148 (1982), 193-214.

[2] M. L. Banner and D. H. Peregrine, Wave breaking in deep water, Annu. Rev. Fluid Mech., 25 (1993), 373-397.

[3] P. F. Byrd and M. D. Friedman, "Handbook of Elliptic Integrals for Engineers and Scientists", Springer-Verlag Berlin Heidelberg New York, 1971.

[4] A. Constantin, On the deep water wave motion, J. Phys. A, 34 (2001), $1405-1417$.

[5] A. Constantin, Comment on "Steep Sharp-Crested Gravity Waves on Deep Water", Phys. Rev. Lett. 93 (2004), 069402.

[6] A. Constantin, The trajectories of particles in Stokes waves, Invent. Math., 166 (2006), 523-535.

[7] A. Constantin, "Nonlinear Water Waves with Applications to WaveCurrent Interactions and Tsunamis", CBMS-NSF Conference Series in Applied Mathematics, Vol. 81, SIAM, Philadelphia, 2011.

[8] A. Constantin, M. Ehrnström and G. Villari, Particle trajectories in linear deep-water waves, Nonlinear Anal. Real World Appl., 9 (2008), 1336-1344.

[9] A. Constantin and J. Escher, Particle trajectories in solitary water waves, Bull. Amer. Math. Soc., 44 (2007), 423-431.

[10] A. Constantin and J. Escher, Analyticity of periodic traveling free surface water waves with vorticity, Ann. of Math., 173 (2011), 559-568.

[11] A. Constantin and E. Varvaruca, Steady periodic water waves with constant vorticity: regularity and local bifurcation, Arch. Ration. Mech. Anal., 199 (2011), 33-67.

[12] A. Constantin and G. Villari G, Particle trajectories in linear water waves, J. Math. Fluid Mech., 10 (2008), 1-18. 
[13] A. Constantin and W. Strauss, Exact steady periodic water waves with vorticity, Comm. Pure Appl. Math., 57 (2004), 481-527.

[14] A. Constantin and W. Strauss, Pressure beneath a Stokes wave, Comm. Pure Appl. Math., 63 (2010), 533-557.

[15] A. Constantin and W. Strauss, Periodic traveling gravity water waves with discontinuous vorticity, Arch. Ration. Mech. Anal., 202 (2011), 133-175.

[16] L. Debnath, "Nonlinear Water Waves", Boston, MA: Academic Press Inc., 1994.

[17] M.-L. Dubreil-Jacotin, Sur la détermination rigoureuse des ondes permanentes périodiques d'ampleur finie, J. Math. Pures Appl., 13 (1934), 217291.

[18] M. Ehrnström and G. Villari G, Linear water waves with vorticity: Rotational features and particle paths, J. Differential Equations, 244 (2008), 1888-1909.

[19] F. Gerstner, Theorie der Wellen samt einer daraus abgeleiteten Theorie der Deichprofile, Ann. Phys. 2 (1809), 412-445.

[20] R. Goyon, Contribution à la théorie des houles, Ann. Fac. Sci. Univ. Toulouse, 22 (1958), 1-55.

[21] D. Henry, The trajectories of particles in deep-water Stokes waves, Int. Math. Res. Not. (2006), Art. ID 23405, 13 pp.

[22] D. Henry, Particle trajectories in linear periodic capillary and capillarygravity water waves, Phil. Trans. R. Soc. A, 365 (2007), 2241-2251.

[23] D. Henry, On Gerstner's water wave, J. Nonlinear Math. Phys., 15 (2008), $87-95$.

[24] D. Ionescu-Kruse, Particle trajectories in linearized irrotational shallow water flows, J. Nonlinear Math. Phys., 15 (2008), 13-27.

[25] D. Ionescu-Kruse, Particle trajectories beneath small amplitude shallow water waves in constant vorticity flows, Nonlinear Anal-Theor, 71 (2009), 3779-3793.

[26] D. Ionescu-Kruse, Exact solutions for small-amplitude capillary-gravity water waves, Wave Motion, 46 (2009), 379-388.

[27] D. Ionescu-Kruse, Small-amplitude capillary-gravity water waves: exact solutions and particle motion beneath such waves, Nonlinear Anal. Real World Appl., 11 (2010), 2989-3000.

[28] D. Ionescu-Kruse, Peakons arising as particle paths beneath smallamplitude water waves in cosntant vorticity flows, J. Nonlinear Math. Phys., 17 (2010), 415-422. 
[29] D. Ionescu-Kruse, Elliptic and hyperelliptic functions describing the particle motion beneath small-amplitude water waves with constant vorticity, Discrete Contin. Dyn. Syst. Ser. B, (2011), in press.

[30] R. S. Johnson, "A Modern Introduction to the Mathematical Theory of Water Waves", Cambridge Univeristy Press, 1997.

[31] H. Kalisch, Periodic traveling water waves with isobaric streamlines, J. Nonlinear Math. Phys., 11 (2004), 461-471.

[32] H. Lamb, "Hydrodynamics", 6th ed., Cambridge University Press, 1953.

[33] H. Lewy, A note on harmonic functions and a hydrodynamical application, Proc. Amer. Math. Soc., 3 (1952), 111-113.

[34] J. Lighthill, "Waves in Fluids", Cambridge University Press, 2001.

[35] V. Lukomsky, I. Gandzha and D. Lukomsky, Steep sharp-crested gravity waves on deep water, Phys. Rev. Lett., 89 (2002), 164502.

[36] A.-V. Matioc, On particle trajectories in linear deep-water waves, arXiv:1111.2490, 1-12.

[37] B. V. Matioc, On the regularity of deep-water waves with general vorticity distributions, Quart. Appl. Math., to appear.

[38] P. I. Plotnikov, Proof of the Stokes conjecture in the theory of surface waves, Dinamika Sploshn. Sredy, 57 (1982), 41-76 (in Russian); English transl.: Stud. Appl. Math, 108 (2002), 217-244.

[39] W. J. M. Rankine, On the exact form of waves near the surface of deep water, Phil. Trans. R. Soc. A, 153 (1863), 127-138.

[40] V. Smirnov, "Cours de Mathématiques supérieures, Tome III, deuxième partie", Mir, Moscou, 1972.

[41] G. G. Stokes, Considerations relative to the greatest height of oscillatory irrotational waves which can be propagated without change of form, Math. Phys. Papers, vol I, Cambridge Univ. Press, Cambridge, 1880, 225-228.

[42] J. F. Toland, Stokes waves, Topol. Methods Nonlinear Anal., 7 (1996), $1-48$.

[43] E. Varvaruca, On the existence of extreme waves and the Stokes conjecture with vorticity, J. Differential Equations, 246 (2009), 4043-4076.

[44] E. Wahlen, Steady water waves with a critical layer, J. Differential Eq., 246 (2009), 2468-2483.

[45] E. Zeidler, Existenzbeweis für permanente Kapillar-Schwerewellen mit allgemeinen Wirbelverteilungen, Arch. Rational Mech. Anal., 50 (1973), $34-72$. 